\title{
Flying through code/space: the real virtuality of air travel
}

\author{
Martin Dodge \\ Centre for Advanced Spatial Analysis, University College London, 1-19 Torrington Place, \\ London, WC1E 7HB, England; e-mail: m.dodge@ucl.ac.uk
}

\section{Rob Kitchin}

Department of Geography, National University of Ireland, Maynooth, Co. Kildare, Ireland; e-mail: rob.kitchin@may.ie

Received 4 April 2003; in revised form 25 July 2003

\begin{abstract}
Commercial air travel is a key global industry facilitating the complex daily movements of planes, people, goods, and services across the world. In this paper we analyse contemporary air travel through the conceptualisation of a culture of real virtuality. We contend that air travel now consists of passage through 'code/space'. Such code/space includes travel websites, check-in, security checkpoints, flight decks, air-traffic control, immigration, and customs checkpoints, which together form assemblages that define the practices and experiences of air travel. Code/space is qualitatively different to coded space, in which software influences the production of space, in that code and space are mutually constituted-produced through one another. This mutual constitution is dyadic so that if either the code or space 'fail', the production of space 'fails'. Our formulation of code/space is nondeterministic and nonuniversal, and how code/space operates and is experienced is embodied through the performances and interactions of the people within the space (between people, and between people and code). In this sense, code/space is constantly in a state of becoming. We illustrate the nature of code/space, and the discursive regimes that support its production, and demonstrate how the code/spaces of an air travel are simultaneously local and global and induce Castells' notions of 'space of flows' and 'timeless time'.
\end{abstract}

\section{Introduction}

Air travel is a key global industry which, since the growth of mass long-haul jet airliners in the 1960s, has become integral to the economic restructuring of globalisation and the emergence of the network society. Air travel provides one of the key physical supports to the global economic system by connecting together people and activities into a shared time and space, whilst simultaneously separating and bypassing other, 'irrelevant', people and places. As the aviation industry has grown in parallel with the network society, it has simultaneously become imbued with its technologies and ethos, increasingly reliant on software engineering and networked computing. Tickets are now bought online, whether individually or through an agent; check-in is automated; security and surveillance rely on sophisticated databases and patternanalysis programs; baggage transfer is sorted by barcode; planes are increasingly flown by computers and guided by software-dependent air traffic control (ATC). In short, the apparatus of air travel from initial transaction to exiting the airport at the final destination is virtualised to the extent that the material transfer of people and goods is dependent on the virtual.

Although we recognise the complex and uneven geographies of mobility produced through the structuring of the airline industry and its selection of routes, the economic geographies produced and sustained by air transport, the elitist nature of air travel, and the social and environmental costs of the aviation industry, this is not our primary concern (see Graham, 1995; IPCC, 1999; Smith and Timberlake, 2001; Weber and Williams, 2001; Whitelegg and Williams, 2001). Instead, our argument is that to make 
sense of air travel, its transactions, mobilities, and developments, it is necessary to acknowledge and understand it, in Castells's (1996) terms, as a real virtuality.

Castells argues that the information revolution of the last couple of decades can be distinguished by the emergence of a new culture-a culture of real virtuality. Here, peoples' daily lives, their experiences are increasingly “... captured, fully immersed in a virtual image setting, in the world of make believe, in which appearances are not just on the screen through which experience is communicated, but they become the experience" (1996, page 373). Castells states; "... the culture of real virtuality [is] where make-believe is belief in the making" (1996, page 375). In other words, it is where the 'real' - "people's material/symbolic existence" (page 373) - is reconfigured and produced through intersections with the 'virtual'.

This new culture is driven by rapid developments both in communications - digital computing, communications networks, and new multimedia systems - and fast, longdistance transport infrastructures-particularly jet travel. In combination these produce radical transformations in space-time relations and facilitate growth in capital and globalisation. Castells captures these radical transformations in the dual notions of the space of flows and timeless times.

The 'space of flows' is defined by Castells as the "... material organization of timesharing social practices that work through flows" (1996, page 412). A flow, according to Castells, is a "... purposeful, repetitive, programmable sequence of exchange and interaction between physically disjointed positions held by social actors in the economic, political, symbolic structures of society" (1996, page 412). It is the space of instantaneous communication and rapid long-distance transport that networks elites-key managers, decisionmakers, and knowledge workers-globally, and the new spaces produced through these flows, for example, gated communities and exclusive resorts. In this sense, the space of flows is the new, dominant, spatial logic, in that the flow-the mobilities, interactions, transactions - that occur through high-speed networked technologies drive the production of capital and create new ways in which capital is spatially fixed (Harvey, 1989). These newly emergent spaces contrast with the more rooted and less fluid nature of the space of places. As a number of critiques of Castells's thesis have noted, however, there are important reciprocal relationships between flows and places with the strength of places acting as powerful 'attractors' for mobile people and capital, so that flows circulate, feed, and sustain places (see Graham and Marvin, 2001).

For Castells, real virtuality also alters the formal qualities of time as processed and experienced, producing a condition of timeless time. Within communication and media technologies, he contends that temporality is erased, suspended, and transformed. It is a "... timeless landscape of computer networks and electronic media, where all expressions are either instantaneous or without predicable sequencing" (Castells, 1998, page 350). History exists only as codes in the networked system, a system that is simultaneously present across time zones around the globe. Moreover, the temporal codes within systems can be split and spliced, so data generated at, or referring to, different times can be recombined in nonsequential forms, inducing a condition of timelessness. Every time a global communication network is accessed and used, timeless time-timelessness and simultaneous presence-is invoked. Real virtuality is "at the same time of the eternal and of the ephemeral. It is eternal because it reaches back and forth to the whole sequence of cultural expressions. It is ephemeral because each arrangement, each specific sequencing, depends on the context and purpose under which any given cultural construction is solicited" (Castells, 1996, page 462). Timeless time evoked by the network society contrasts starkly with other forms of time, for example 'natural' time (for example, Earth seasons, diurnal cycles, body clocks), 'social' 
time (for example, national holidays, celebrations, festivals, holy days), and also the regular, predictable 'clock' time of the industrial age.

In the rest of this paper we explore how air travel has become a real virtuality par excellence, seamlessly blending the material (travel) and virtual (networked communication) - the two keys to Castells's thesis - to produce new spaces that facilitate the space of flows and timeless time. These new spaces we call 'code/spaces' - spaces in which the materiality of air travel is produced through information and communications technologies (ICTs) and software systems, which are themselves produced through spatial mobility (they are created by interactions and transactions). Our argument is that these code/spaces work to produce a totalising aviation environment - a complex set of interlocking assemblages - that define the practice and experience of air travel. This is in marked contrast to most other everyday spaces, which we call 'coded space', where software systems and processes contribute, but do not dominate, the production of spatiality. In the next section we define code and elaborate our notion of code/space. This is followed by an examination of the code/spaces of aviation and a discussion of the production of code/spaces and the discursive regime that supports and deepens their pervasiveness. In the final section we outline how code/spaces (re)produce the space of flows and timeless time.

\section{Defining code and code/space}

"More and more ... the spaces of everyday life come loaded up with software, lines of code that are installing a new kind of automatically reproduced background and whose nature is only now starting to become clear."

Thrift and French (2002, page 309)

At its most fundamental we define a code to be an instruction or rule that has a single outcome determined by binary logic (yes/no). The combination of these individual logic rules produces codes (programs) that are capable of performing complex functions. Here we are concerned with a particular class of codes, these being computer software. Software is multiple in form and includes hardcoded applications with no or limited programmability (for example, embedded chips in aircraft systems or security sensors), specialised applications (for example, air-traffic control systems, baggage-routing programs, banking software), generic applications (for example, word processing, spreadsheets, web browsers), and operating systems (for example, Windows, MacOS, Unix, Linux). These software run on a variety of hardware (for example, embedded chips, dedicated units, PCs, workstations) and can distribute, generate, monitor, and control data exchange and flow across a range of infrastructures (for example, ethernet, fibre-optic cable, wireless, satellite) using a variety of forms (for example, electrical, light, microwave, radio). The coding itself within these programs varies from abstract machine code and assembly language to programming languages, applications, macros, and scripts. The extent to which these various forms of software matter is, we suggest, a function of autonomy and consequences. Here, autonomy relates to the extent to which code can do its 'work' without direct human oversight or authorisation, with this varying with inputs (knowledge of its environment), sophistication of processing, and range of outputs. If code fails, then its consequences can range from mild inconvenience through serious economic and political impacts to life-threatening situations.

The power of many software applications derives from their use of data held in relational databases (databases with common fields that allow them to be easily married and interrogated). Indeed, all aspects of air travel-the passengers, their baggage, the planes, and the crews - are encoded in various systems as data, and 
their physical journey traced and regulated by the transmission of data through virtual space. This is an aspect of the ongoing and deepening processes that abstract information from, and use networked infrastructure to regulate, materiality, seen elsewhere in the 'information society' most notably studied in relation to the planning and management of city spaces (for example, Batty, 1997a; Graham and Marvin, 2001; Mitchell, 1996).

Rather than code and its attendant data merely circling geographic space in a parallel world of their own, with virtual and material fleetingly intersecting, we contend that the materiality of air travel and its software and data have become mutually constituted - produced through one another. Progress from buying a ticket, to moving through an airport, to travel on a plane is mediated through code/space-space produced through code. These aviation code/spaces, we believe, are qualitatively different from most other built environments that are monitored and regulated by spatial databases and software supported by networked infrastructure, such as city streets with closed-circuit television (CCTV), traffic-management systems, and so on. In the case of aviation, the relationship between code and space is dyadic-code and space are mutually constituted, wherein how the space is used and produced is predominantly mediated by code, and the code and its data exist in order to produce the space and its attendant spatiality. This relationship contrasts with the more ambivalent or symbiotic association between networked databases/technology and city streets, which we would consider as 'coded spaces', or as Batty (1997b) puts it 'cyberplace'-spaces that are digitally surveyed and regulated, but where the relationship between code and space is not mutually constituted. In other words, in code/space, code dominates the production of space, explicitly mediating sociospatial processes and experience- "Software quite literally conditions ... existence" (Thrift and French, 2002, page 312), whereas in coded spaces, code merely contributes in some way to the production of space.

The distinction between coded space and code/space is not just a matter of the amount of code (in terms of the number of lines of code, or the density of software systems). In a code/space, the domination of code is so pervasive that if one half of the dyad is put out of action then the entire code/space fails (for example, a security alert closes an area, or a system crash closes a check-in area, or a failure in a plane system grounds an aircraft). Here, there are no alternatives: check-in agents are not trained or authorised to do manual check-in and the destination airport would not accept the passengers; planes cannot fly without many crucial digital systems that lack manual counterparts; ATC system failure will ground all planes-again because manual systems are insufficient or have been discontinued; for example, the 'crash' of the Tokyo Air Traffic Control Center on 1 and 2 March 2003 meant the cancellation of over 203 flights (Risks List, 2003). In other words, the production of spatiality within code/space is dependent on its dyadic nature. In coded space, code matters to the production and functioning of a space but if the code fails the space continues to function as intended, but not necessarily as efficiently, safely, or with as little cost. Here, the role of code is often one of augmentation, facilitation, monitoring, and so on, rather than control and regulation. The dyadic nature of code/space can be illustrated both in relation to passage through an airport and flight itself.

\section{Code/spaces of air travel}

In the airport, check-in is verified by unique passenger and ticket codes; progress from check-in to plane door is sanctioned by surveillance codes and security checks; routes through the airport are guided by flight codes, departure-gate numbers, terminal numbers displayed and updated on monitors; baggage is routed through barcodes and tags. At the flight destination we enter another set of code/spaces: immigration, 
controlled through personal identification codes (for example, passport number); routes to the correct baggage carousel; signage in the arrivals hall; and so on. Much of this code/space is largely routinised and backgrounded (become part of the everyday), and many aspects remain invisible or unexplained. This is easily overlooked or dismissed as banal (Parker, 2002), or accepted as unproblematic and 'natural' ('just the way the system works'); as Thrift and French note, “... even though software has infused into the very fabric of everyday life - just like the automobile - it brings no such level of questioning in its wake" (2002, page 313). Yet there are quite clear political questions in relation to such ambivalence (though these are beyond the scope of this paper).

A passenger ticket is in many ways a material embodiment of code/space (see figure 1 , over) on which are printed several data codes, which although meaningful to the software programs that facilitate travel, are mostly meaningless to the passenger. With the move to e-tickets, these codes are often hidden further: reduced to a single unique code number that identifies the passenger at check-in. The prime purpose of these data is to verify that the ticket holder and ticket match and that the person is cleared to progress to the next stage as they move through the airport and on to their destination. Many of the invisible codes refer to comprehensive customer-management systems maintained by airlines that profile passengers based on their frequent-flyer status, ticket class, and so on, or other forms of security-profiling systems such as those that are increasingly being implemented to identify the 'guilty' (or potentially guilty) from the 'innocent' (see Lyon, 2003). A prime example of the latter is the US system known as CAPPS (computer assisted passenger prescreening system), which uses routine transaction information (for example, how a ticket was paid for) to identify 'suspicious' activity. This system is being significantly enhanced post September 11 in terms of the range of data inputs and pattern analysis (Lyon, 2003; Salkever, 2002). The aims of these code/spaces are not just to describe and model but in some senses to simulate and actually predict passengers' behaviour (Graham, 1998). Both of these systems - for customer and security profiling - potentially have material consequences for passage through the airport and flight, for example, providing upgrades and access to airline lounges, or targeting for special security checks and questioning.

Indeed, one of the prime uses and effects of code/space in the airport is the creation of a panopticon which enables and enforces a regulatory environment in which passengers (and most staff) are rendered, in Foucault's (1978) terms, docile bodies: bodies that pass through the system in an orderly, noncomplaining, compliant manner. Here, code/spaces discipline through visible systems of detection (for example, unique identification check-in and immigration, surveillance cameras, security checkpoints), in addition to uniformed personnel, and accompanied by stiff sanctions (for example, additional searches, fines, termination of a journey, criminal charges). ${ }^{(1)}$ In this sense, following Lessig (1999), code/spaces are regulatory spaces where 'code is law'. Unlike ordinary law, however, code law is often more ambiguous, inflexible, and nontransparent for reasons of security or commercial confidentiality, and is more difficult to appeal.

Similarly, the plane itself is a code/space from the cockpit through to the in-flight entertainment system and digital maps displayed in the cabin. Over the past twentyfive years or so avionics has dramatically changed flying for pilots. The increasing

(1) The maintenance of docile bodies is continued on board the plane through safety briefings, seat belt warning lights, rules, and regulations (for example, limited or no use of electronic equipment and mobile phones), meal and entertainment service, all overseen by cabin staff. This leads Crang (2002, page 573) to note that "... the actual experience of flying is one of the most infantilised environments possible." 
N: PASSENGER TICKET AND BAGGAGE CHECK

Issued Br britishmidlande

ECONOMY

FLIGHT COUPON 1 OF 2

PASSENGER COUPON

DOME OF PASSENGER MOT TRANSFETABLP

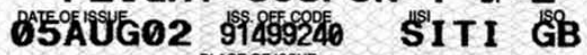

DODGE/MARTIN MR

WCOOL

DISC TOUACOOE

LHR 1 BDD

DUBLIN

EONONDON

LHR 1

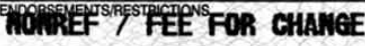

ORIGINAL ISSUE

ISSUED IN EXCHANGE FOA

YELKRPCOOE $/ 1 \mathrm{~A}$

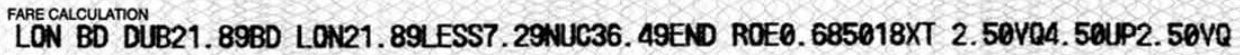

FARE

GBP25.00

EQUN. FAREPD.

Firi

BUBLIN

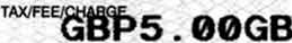

PCS. CK WT. UNCK. WT

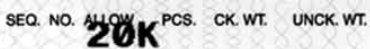

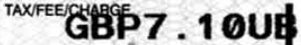

TAXFEE GHAG $9.50 \times 723600284162233$

TOTAL GBP46.6C

$12362128907832 \quad 4$

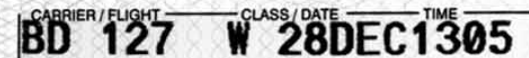

GATE BOARD TIME SEAT SMOKE

ADOMIONAL SEATINFORMATION

PCS. CK.Wt. UNCK.WT. SEQ.No. PCS. CK.WT. UNCK.WT. BAGGAGE ID NO.

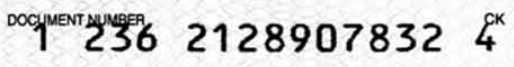

Figure 1. A typical airline ticket displaying numerous different codes (source: authors). 
integration of sophisticated virtual geographic representations into the cockpit means that pilots now fly through 'real space' virtually, using digital instruments [artificial horizons, inertial and GPS (global positioning satellite) navigation], radio communications systems, real-time radar map displays, and so called 'fly-by-wire' controls, collision-avoidance systems, with continuous feedback from onboard sensors and ground-based data streams (see figure 2). The Boeing 747-400, a leading long-haul jet aircraft, has some 400000 lines of software code to power its numerous cockpit avionics systems (see table 1, over); the next generation Boeing 777 aircraft has some 79 different computer systems requiring in excess of 4 million lines of code (Pehrson, 1996). The degree to which flight is mediated by code is even greater in the case of military aviation where the pilot is supplemented by advanced computer tactical systems, electronic warfare countermeasures, head-up displays, night and infrared vision, or even replaced by code, for example, in the case of unmanned aerial vehicles such as the

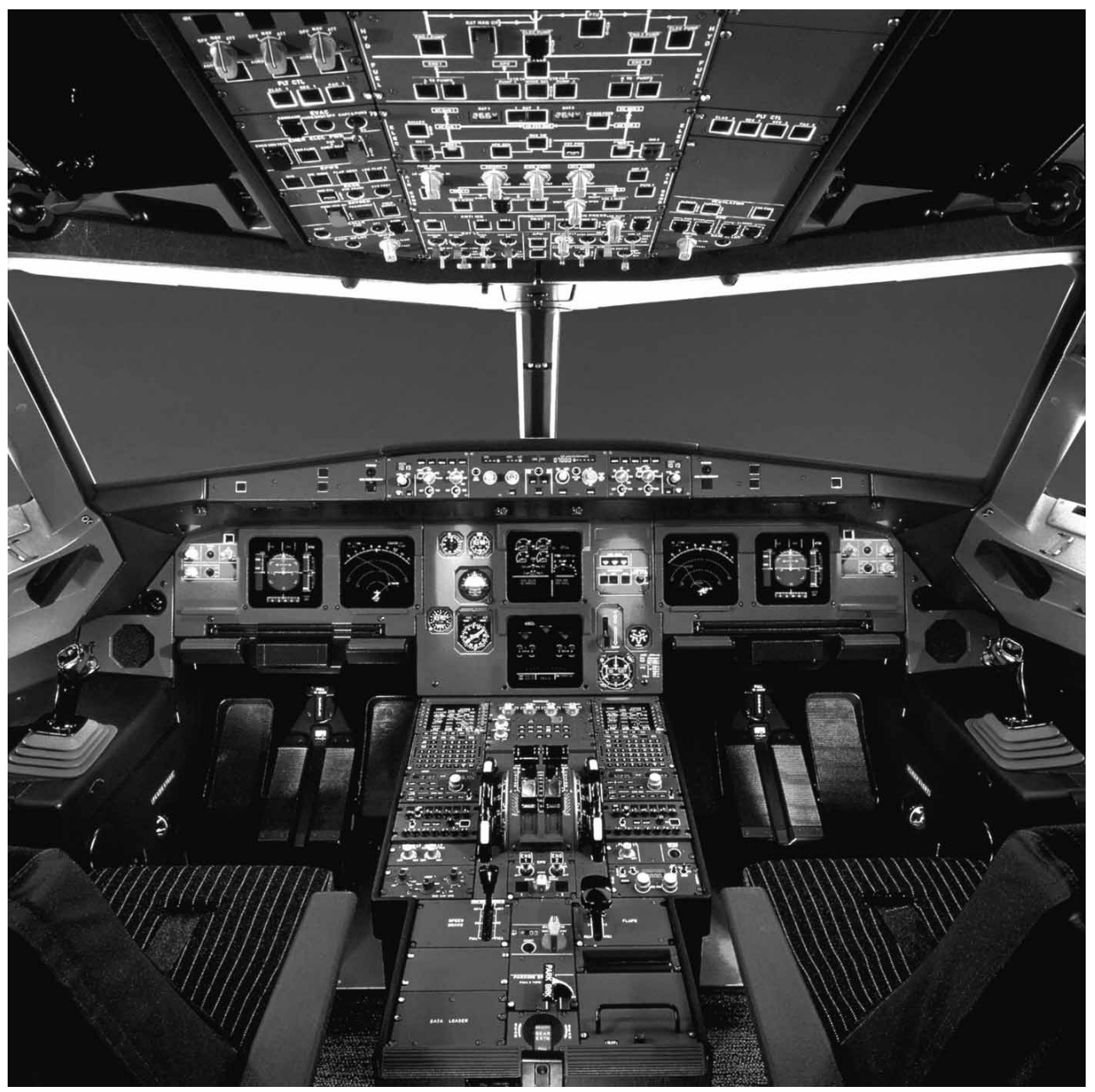

Figure 2. View of the cockpit of an Airbus A320 airliner which shows the numerous digital displays and control systems. The two screens in front of the pilots provide output from the Electronic Flight Instrument System, while the middle two screens show data generated by the Electronic Centralized Aircraft Monitor. This environment is the epitome of real virtuality. (C) Airbus, 2003. Source: http://www.airbus.com/dynamic/media/photo.asp). 
Table 1. Major avionics systems in the Boeing 747-400 airliner (source: http://www.boeing.com/ commercial/747family).

Flight deck voice recorder

Weather radio transceiver

Instrument landing system (ILS) receiver

Airplane condition monitoring system

Central maintenance computer

Inertial reference unit

ATC (air traffic control) transponder

VOR (VHF omni-range) receiver

Selcal (selective calling) decoder

Central management unit

ADF (automatic direction finder) receiver

ATC control panel

TCAS (traffic collision avoidance

system) processor

Indicator standby airspeed

Flight data recorder
Flight control computer

Air data computer

GPWS (ground proximity warning systems)

HF communications receiver

Flight management computer

DME (distance measuring equipment)

interrogator

ACARS (aircraft communications addressing and reporting system) management unit

Audio management unit

EICAS (engine indication and crew alert system) interface unit

Weather radar panel control

Control display unit

Infrared mode selector

Indicator standby attitude

CIA's Predator plane. Further, pilot and plane performance is continuously monitored and recorded by so-called 'black boxes' and pilots are trained in sophisticated flight simulators (see MacPherson, 1998; Rolfe and Staples, 1986).

Moreover, flight takes place through the code/space of the atmosphere. Here, the space is coded by ATC systems that direct planes between destinations. Developed after World War 2, these systems such as the early SAGE (Semi-Automated Ground Environment) air-defence system developed for Pentagon by IBM in the 1950s, virtualise material space (see Edwards, 1996; Everett et al, 1983) (see figure 3).

Taken to an extreme, the code/spaces of air travel extend to the Internet and the systems through which tickets are purchased (for example, travel websites, booking databases, credit-card encryption) and global financial markets (the networked spaces of banks, stock markets, financial districts, insurance centres) that, with the recent volatility in the airline industry after September 11, play a large role in defining airline viability and in restructuring routes and services.

Another way in which these code/spaces are revealed is when the production of space fails, either through minor 'glitches' or, rarer, catastrophic incidents. Examples of glitches are when luggage is 'lost' (misrouted) or passengers are 'bumped off' of a flight because of overbooking. Interestingly, in these cases it is common for airline staff to be at a loss to explain the cause, simply blaming 'the computer' for the problem. Bumping is, however, not a system error, but the logical outcome of commercial pressures wherein complex (proprietary) yield-management systems work to maximise profits by ensuring that planes fly as full as possible. These systems rely on notional behavioural rules and statistical models of passengers, which assume that not all reserved passengers will actually turn up and want to fly. The very survival of an airline can depend on how well its yield management is working as every empty seat is revenue lost. In terms of catastrophic accidents, the cause of many incidents which have been traced to so-called human error are more often a result of complexity of technical systems and breakdown in the human-computer (code/space) interactions, particularly for pilots (see Baberg, 2001).

Airline delays and flight cancellations also highlight the real virtuality of airlines. Once ordered schedules begin to unravel, perhaps because of ATC problems or bad weather, then the smooth operation of the airport can quickly be disturbed. As the in-and-out flows of passengers become interrupted, the airport environment quickly changes character, with crowds of angry passengers and, at the worst times, stranded 


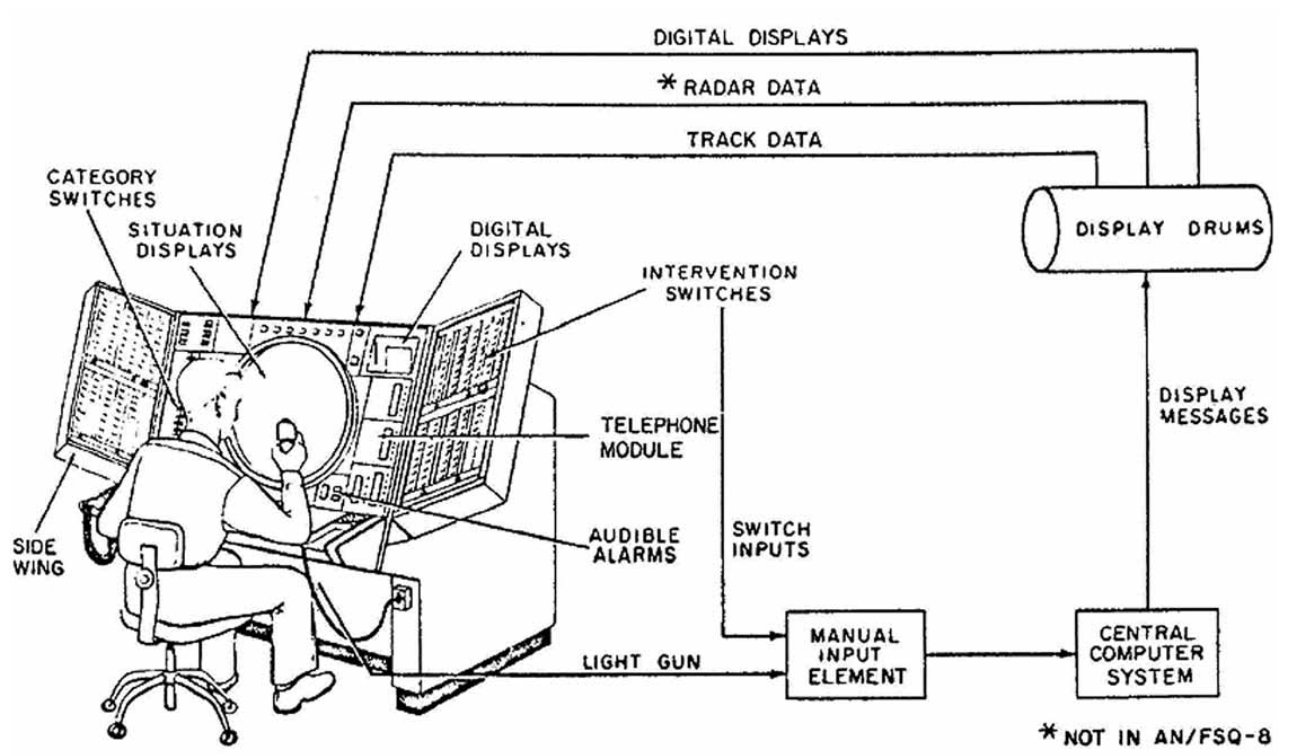

Figure 3. Schematic of a 1950s SAGE air defence system operator's terminal showing the various data inputs. It illustrates how the human operators are meshed within code/space as far back as the 1950s. (Source: scanned from an original SAGE manual, http://ed-thelen.org/Sagelntro.html, courtesy of Ed Thelen.)

people forced to sleep in departure lounges. The worst of these breakdowns in the space of flows make news headlines, with reporters making typically doom-laden pronouncements of 'travel chaos'.

It is important to note that the code/spaces of air travel have not been planned in a comprehensive manner, but rather have accreted over time with technological advances, and political and economic decisions to employ such technologies, to create vast interlocking assemblages of code/spaces. The components of these assemblages have a diverse range of owners, maintainers, and licensing agreements that parallels the labyrinth layers of contracts, leasing, and service-level agreements that characterise the industry. Further, a raft of national and international bodies and industry organisations are responsible for the setting and vetting of standards for systems where code is vital, particularly in relation to safety standards and reliability for real-time critical systems like aircraft navigation and ATC. Moreover, these complex assemblages - ticketing, security, surveillance, flight, traffic control, immigration, and so on-are relational, not discretely referenced to individual passengers and airports but, rather, stretched out across the whole architecture of networked infrastructure of air travel from the locations from where tickets are initially purchased to final destination. Code/spaces are simultaneously local and global, grounded through the passage of people and goods, but accessible from anywhere across the network; and linked together into chains that stretch across space and time to connect start and end nodes into complex webs of interactions and transactions. As accretion and interconnection continues apace this is deepening the impact of code/space, strengthening the dyadic relationship between code and space, and the power of code/spaces as regulatory systems is multiplied. These assemblages, then, have no central control and a complexity much greater than the sum of the parts (the individual software and hardware components). Consequently, the case could be made that code/spaces of aviation need to be analysed (1) as an assemblage that is, in Deleuze and Guatarri's 
(1987) terms, striated - that is, complex, gridded, hierarchical, rule-intensive, regulated; and (2) as a complex system with emergent properties (Holland, 1998; Waldrop, 1994).

\section{Producing code/space}

Although this formulation of code/space - space produced through code-smacks of technological determinism (for example, codes determine absolute, nonnegotiable ways the production of space and the sociospatial interactions that occur within them) and universalism (for example, that such determinations occur in all such spaces and at all times in a simple cause-and-effect manner), these are charges we would refute strongly. It is true that within code/space the nature and meaning of space is dyadically produced through code, so that this mutual constitution makes a difference to the production of space, defining the practice and experience of air travel. This is most obvious in the context of air travel with regards to regulation, for example defining explicitly who has access to each code/space within the chain of assemblages - whether traveller or worker-and what is acceptable and expected behaviour within these arenas (with associated penalties such as warnings, expulsion, fines, and imprisonment). Here, the mutual constitution of code and space is pervasive, consistent, routinised, effective (action is instigated if the 'rules' of code/space are violated), and determinate (but only in the sense that, as noted, failure of either code or space means that code/ space fails and the intended production of space is not produced). That said, how code/space actually operates and is experienced is open to rupture: code/space is embodied through the performances and interactions of the people within the space (between people, and between people and code). In this sense, code/space is not consistently produced, always manufactured, and experienced the same. Instead, code/ space is constantly in a state of becoming, produced through individual performance and social interactions that are mediated, consciously or unconsciously, in relation to the mutual constitution of code/space. This can be illustrated in a number of ways.

First, the extent to which space and code are mutually constituted and their effects explicitly invasive - where code/space is visible and explicit in its consequences - alters as one passes through the assemblages of air travel. For example, within transition zones characterised by the intersection of flow and security (for example, check-in, security checkpoints, boarding, immigration) the visible production of code/space is magnified so that a highly particularised production of space is relatively consistently produced through the explicit scrutiny of every inhabitant (passenger or worker). Whereas in the check-in hall, or the departures lounge, or airport shopping mall, or the walkway from lounge to gate, or in the air, or baggage-reclaim area, the mutual constitution of code and space is backgrounded and less invasive unless provoked. For the most part, then, except for the small proportion of time spent in these transition zones, the vast bulk of time in code/space-either in the airport or in the air-is largely banal: spaces of chat, gossip, waiting, shopping, fidgeting, reading, staring, eating, and so on (see Gottdiener, 2001).

Second, even within the more invasive transition zones, code/space is still negotiated. Whereas the processing of passengers is verified and approved by code, the decision to allow onward passage through the assemblages is often mediated by an airport or airline employee (for example, ticket check-in agent, check-in supervisor, security guard, flight attendant, immigration official, customs officer). These individuals are often vested with the powers to disregard and override 'the system', for example allowing passage without full documentation or passage through security after setting off of alarms. (At other times the staff can also choose to not override the system for professional, commercial, or even personal reasons.) 
Third, it is clear from observation that code/space is experienced differentially - not everyone experiences code/space in the same way (and not simply on the basis of privilege). For example, although we would argue that a plane is a code/space, it is experienced by crew and passengers very differently (as is every assemblage), but not in a binary way (all passengers and all crew have the same experience). Rather, code/ spaces are peopled and despite theses that largely empty airports and air travel of culture and meaning [so-called 'nonplaces' (Augé, 1995)] the identity politics that sustain divisions in labour and between passengers still operate (for example, factors such as class, gender, and race still shape social interactions) (Crang, 2002). This is not to say that code/space does not facilitate homogenisation of built and regulatory environments: rather, it is to acknowledge that airports are diversely peopled, and that the meanings and experiences of air travel differ precisely because of how they are peopled.

Fourth, code/spaces are open to subversion. Here, the intended purpose of the code/space - security, safety, immigration, and so on - is subverted through the 'system' being duped: for example, people illegally passing through immigration, or goods bypassing customs, or more exceptional cases such as hijacking and bombing. In this sense, although the system strives for perfection in terms of regulating and producing code/space, it continues to have cracks that allow through unintended people, goods, or acts.

All of these examples mean that the nature and production of code/space are never fixed, but shift with place, time, and context (for example, social, political, and economic). As such, understanding the everyday production of code/space requires an analysis of how code/space is performed-as embodied practice within a coded regulatory environment - rather than enforced in some deterministic fashion. Of course, this coded regulatory environment does not exist in isolation and is itself reproduced through a discursive regime.

\section{The discursive regime of code/space}

For Foucault (1977), a discursive regime is a set of interlocking discourses that sustain and reproduce through processes of definition and exclusion, intelligibility and legitimacy, a particular set of sociospatial conditions. In other words, a discursive regime provides the rationale for how space is predominantly produced, legitimating the use of discursive and material practices that shape its production. The discourses that constitute a discursive regime work to promote and make commonsense their message, but also to condition and discipline: their power is persuading people to their logic; to believe and act in relation to this logic. For example, in relation to sexuality, Foucault (1978) documents how interlocking discourses including medical theory, religious doctrine, and political rhetoric work together to create a discursive regime that has promoted and justified the policing of familial heterosexuality as the most natural, normal, and moral form of sexuality, and has persuaded individuals of this logic and to discipline their own sexual behaviour accordingly.

The code/spaces of air travel and their regulatory power is similarly supported by at least five interlocking discourses that persuade and discipline passengers and workers through their logic. These are security, safety, economic rationality, convenience, and 'free skies'. (2) These discourses work ideologically to construct and position

(2) These are not the only discourses that shape the airline industry. For example, much of the airline industry has been intimately tied to national politics as state-owned (or state-subsidised) flag carriers are seen as strategic national assets and vehicles for state image building (Sampson, 1984). As such, much of the airline industry is shaped by nationalistic concerns (Butler, 2001; Van Zandt, 1944). 
code/space as commonsense: it benefits passengers and workers, society and the aviation industry alike, with few negative costs (and these are more than outweighed by benefits). We take each of these in turn.

Airports have been and continue to be perceived as targets of terrorism, they are a means of international travel and smuggling for terrorists and criminals, and planes are involved in accidents. Code/space, it is argued, is desirable because it creates a more secure and safe environment. Code/space allows the surveillance of passengers and workers to become more panoptic in scope, both widening and deepening the extent to which air travel can be policed and thus made a more secure and safe undertaking. The rationale of this discourse has, of course, been strengthened after September 11, with fears of global terrorism being mobilised to justify increases in the density and intensity of security checks and the implementation of more invasive surveillance and predictive systems, such as the development of the CAPPS II passenger profiling in the USA (Lyon, 2003; Salkever, 2002). Part of the power of code/space here is that it is what Foucault calls a 'technology of the self': a sociospatial configuration where the presence of the technology persuades people to self-discipline their behaviour; to act in ways prescribed by those controlling the space and technology. In the case of airports this is reinforced through regular security warnings about unattended baggage, check-in security questions, warnings not to tamper with the toilet smoke detectors, and so on. Safety is not always intimately tied to security, and another set of discourses promotes code/space as a way to ensure safe passage. For example, code/space ensures effective air-traffic control, reduces demands on pilots, provides a means of remote detection of and automatic response to system failures, and so on. Further, airlines stress their maintenance record and aircraft manufacturer's competence. These discourses of security and safety are powerful because they are designed to enhance trust and confidence in the air-travel industry. Code/space is positioned as the foundation for confidence by providing 'infallible' systems. It should be noted that these discourses of security and safety are further reinforced by government regulations, legislation, and international treaties (see Butler, 2001; Graham, 1995; Van Zandt, 1944).

Another set of discourses justifies the production of code/space by contending that it creates a more convenient or cost-effective journey. Code/space reduces the hassles of flying. An individual can book online, meaning they do not have to visit an agent; they can pass smoothly and efficiently through the airport, the systems automatically acknowledging status such as frequent flyer, providing upgrades, and so on. Code/ space also enables passengers to stay in contact with the space of places through, for example, access to phone and e-mail in-flight. Code/space also reduces costs both for passengers and for airlines. The rhetoric produced contends that by automating aspects of the industry, airlines can reduce their fares and pass on savings to customers, while at the same time increasing their profits (a win-win situation). For example, online booking means the airline does not have to pay an agent; automatic baggage sorting reduces the need for baggage handlers; automated surveillance reduces manual surveillance; and so on. Further, code/space is economically rational because it facilitates global trade and tourism, creating and sustaining wealth creation (Castells, 1996). Here, code/space seduces people to its logic. In Althusser's (1971) terms, code/space interpellates by enticing people to subscribe to and desire its logic and to participate willingly and voluntarily in its ideology and practice (rather than simply disciplining them into docile bodies). 
This interpellation is key to another discourse: that of 'free skies'-one that paradoxically undermines security discourses. (3) The ethos of air travel, from its very beginnings, has been one of 'freedom of the air' and 'open skies'. Implicit in the rhetorical messages of aviation is the deeply utopianist logic that 'making the world a smaller place', will 'make the world a better place'. Air travel is presented as a benevolent force that is inherently good for commerce and can bring greater understanding between people. The airline industry strives to portray an image that transcends the geopolitics of the terrestrial world and offers an uncomplicated world without frontiers. The commercial aircraft manufacturers and airlines spend heavily on marketing and advertising to promulgate this powerful, idealistic rhetoric, often utilising universalist visual tropes of the globe and world route maps (Cosgrove, 1994; Fleming, 1984). Of course, code/space is now promoted as an integral part of making such utopian rhetoric a reality.

As Foucault (1977; 1978) noted, a discursive regime does not operate solely from the top downwards, but through microcircuits of power - the outcome of processes of regulation, self-regulation, and resistance. As such, passengers and workers within airports are not simply passive subjects, disciplined and interpellated in linear and unproblematic ways by the discourses we have just outlined. Rather, such discourses are open to rupture: subversion, resistance, and transgression (for example, cases of 'air-rage'; environmental protests against airports; anticorporate websites highlighting the iniquitous practices of global airlines, such as http://www.untied.com/). In this sense, power is not captive, purely in the hands of a few, although the discursive regime operates - in conjunction with the operation of code/space - to produce such a hegemonic order. In other words, code/space and its discursive regime work simultaneously to reinforce and deepen their logic and reproduction, at the same time as others seek to undermine, resist, and transform their hegemonic status. Although undoubtedly the aviation industry and its attendant code/spaces have detractors, it is fair to say that, at present, code/space is the hegemonic production of space associated with air travel. This, we contend, creates and reproduces a condition of real virtuality, which in turn helps to sustain the rhetoric and use of code/space.

\section{Code/space, space of flows, and timeless time}

As noted, our contention is that the virtual transformation of the aviation industry to one that is reliant on code/spaces sustains and reproduces Castells's notion of the culture of real virtuality and its attendant space of flows and timeless time. Air travel is a central component of the new spatial logic of the space of flows, allowing the rapid transit of transnational elites across the globe. Air travel fulfils Castells's conditions because it works at three different 'scales': material, logical, and social. First, the aviation industry consists of a well-developed material infrastructure, specifically designed to facilitate rapid and efficient transport of people, goods, and data. This infrastructure consists of airports, planes, air-traffic control, air routes, information technology systems, data networks, and so on, attendant infrastructures such as fuel, food, hotels, on-ground transport links, highly organised to maintain throughput including flight, maintenance, and staff schedules. These networks are stretched out across the globe, both through the infrastructure itself and through the complex commodity chains that provide inputs necessary to allow the industry to function.

(3) The incessant drive for economic rationalisation can create a tension with regards to the costly endeavours of security and safety. Indeed, cost cutting with regards to passenger screening through the use of deregulated, nonunionised, private, security staff was one of the chief foci of blame for September 11 . 
Code and data, we would argue, are the vital 'glue' that keeps these highly complex systems tied together and functioning.

Second, the aviation industry has a logical hub-and-node structure that maximises the functioning of the system by organising it hierarchically, both locally and globally, recognising that the space of flows is not evenly spread across the globe but is, rather, concentrated at certain locales. Third, the aviation industry to a large degree grew out of its function to enable rapid and efficient movement of dominant, managerial elites across the globe in order to manage transnational concerns, and this continues to be one of its primary functions despite the rise of low-cost and charter flights. This is manifest in the architecture and spatial practices of the airport through special channels at check-in, executive lounges, expensive designer shops, segregated seating on planes, five-star hotels and conference facilities near to airports, and so on.

Further, many aspects of air travel evoke Castells's notion of timeless time. The aviation industry, we would argue, generates its own time-space, which takes precedence over 'natural' times of the body, diurnal cycle, seasonal variation as well as conventional, grounded, local 'clock' times of the space of places. In this sense, we argue that air travel is an 'out of time' experience. This can be illustrated in three ways. First, the major airlines operating global routes function on a twenty-four hours a day, seven days a week, basis and their schedules, flights, staffing, and ground operations can be thought to have created their own time-space, stretched out and shared across all the places they interconnect, and which is distinct from the 'natural' rhythms of time in those locales. Passengers have to 'fit into' this time-space, reorganising their schedule so that it parallels the airlines. Similarly, airline and airport staff have to work to (unsociable) schedules that are designed to facilitate flights landing or taking off in different time zones, and residents close to airports are subject to the noise pollution from planes operating at a disjuncture with local time.

Second, air freight has grown enormously, with the promise of next-day delivery to nearly anywhere in the world (for a price). This has led to massive space-time compression in the transfer of high-value goods and documents. Here, the timeless time of aviation means that, for the Western consumer at least, a 'permanent global summertime' exists with regards to goods such as exotic fruit, vegetables, and flowers. That said, the vast majority of goods continue to be transported across the space of places by road and container ship. To use a different example, contagious diseases can be spread extremely rapidly, negating slower diffusion across the space of places. This is well illustrated by the spread of new forms of pneumonia, such as SARS (see Bradsher, 2003), as infected passengers can cross continents by plane before symptoms manifest themselves (Yach and Bettcher, 1998).

Third, the airline industry is regulated through time defined by specific safety regimes that dictate how long a pilot may fly, or how long an aircraft can remain in service without maintenance. The nature of timeless time is perhaps most apparent for passengers through the experience of 'jet lag', caused by the disturbance of the body's internal 'body clock'. Here, the rapidity of global air travel and having to adapt to a new time zone warps the natural biological rhythms and cycles of the body, most particularly in terms of waking, sleeping, and eating.

Important to note here is that the production of the space of flows and timeless time, in turn, reproduces the value of code/spaces, ensuring that more and more aspects of air travel become virtualised. This in turn recursively reproduces the development of the space of flows and timeless time. In other words, code/space and real virtuality are also produced through each other. 


\section{Conclusion}

Code is the lifeblood of the network society, just as steam was at the start of the industrial age. Code, like steam, has the power to shape the material world; it is able to produce space. Code has enabled the air-travel industry to transform the way in which it is organised, managed, and operates. It has radically reconfigured work practices within the industry and transformed the experience of travel. It has led to an industry that is more effective in its disciplining and interpellation of passengers and staff. This success is, we have argued, a result of the dyadic relationship between code and space in air travel, with code a vital component in booking tickets, securing passage through an airport, enabling flight, and so on, thus creating a complex assemblage of code/spaces. These code/spaces are produced through the mutual constitution of code and space, wherein the material and virtual are produced through each other. In other words, in the case of code/space, how space is used and produced is predominantly mediated by code, and the code exists in order to produce space. Indeed, such is the dyadic power of code/space within the aviation industry that we contend that the failure of the code leads to a failure of the intended production of space and that part of the aviation system grinds to a halt with no alternative means of producing the intended actions (for example, the throughput of passengers). Code/space is thus dominant in defining the practice and experience of air travel.

That is not to say, however, that our formulation of code/space is deterministic or universal. Instead, the way code/space operates and is experienced is embodied through the performances and interactions of the people within the space (between people, and between people and code). In this sense, code/space is constantly in a state of becoming, produced through individual performance and social interactions that are mediated, consciously or unconsciously, in relation to the mutual constitution of code/space and its supporting discursive regime. In this sense, for the most part code/space is experienced ambivalently or banally. Further, code/spaces are simultaneously local and global, grounded through the passage of people and goods, but accessible from anywhere across the network; and linked together into chains that stretch across space and time to connect start and end nodes into complex webs of interactions and transactions. As such, they should be understood and conceptualised as relational spaces.

The prevalence and rationale for the pervasiveness of code/spaces within the aviation industry is, we contend, reproduced through a sophisticated discursive regime composed of at least five interlocking discourses: security, safety, economic rationality, convenience, and 'free skies'. These discourses work together ideologically through discipline and interpellation to promote the development and use of code/space as commonsensical and rational, benefiting passengers, the airline industry, and wider society, with few negative costs which are more than outweighed by benefits. Although these discourses are opposed, they presently remain hegemonic in status.

Indeed, it is clear that code/spaces are an integral aspect of the aviation industry, with industry operations now so complex and interdependent that they can only be managed through the use of code. As such, it is extremely unlikely that there will be regression back to more manual systems and it is inconceivable that new generations of planes or new airport terminals will be constructed with less coded systems and spaces. Instead, the trend to create code/space and to deepen the relationship between code and travel has been accelerated with the enhanced security concerns after September 11, the desire for technical fixes, and the fact that highly coded environments tend to attract more code. This means that code is likely to become increasingly central to the management and operation of the air industry, especially in relation to security, safety, and augmenting flight. This is clearly going to reduce the 
number of workers as a ratio of passengers, reduce and redefine the role of those managing the infrastructure and services, and increase the panoptic and regulatory control of air travel. Further, key elements of air-travel code/space (such as customermanagement and security-profiling systems) are starting to be replicated in other commercial and state arenas, often utilising spatial technologies such as GIS (see Curry, 1998; Graham and Wood, 2003).

In turn, we contend that these code/spaces constitute a real virtuality which, in the case of air travel, supports Castells's notion of the space of flows, creating a complex networked material and virtual infrastructure stretched out across the globe through which managerial elites and data travel; and timeless time, wherein this infrastructure creates a novel time-space configuration, simultaneous presence, and timelessness. This condition of real virtuality, in turn, reproduces the need for code/space and thus deepens its logic and fosters its growth in a recursive relationship.

The aviation industry, perhaps more than any other, demonstrates the salience of code/space. We would suggest, however, that the concept also has utility for understanding other instances where code and space are mutually constituted, such as financial trading rooms and call centres. These code/spaces and their relationship to coded spaces and spaces devoid of code needs further examination.

Acknowledgements. We are grateful to the helpful comments provided by Sean Gorman and the three anonymous referees.

\section{References}

Althusser L, 1971 Lenin and Philosophy and Other Essays translated by B Brewster (New Left Books, London)

Augé M, 1995 Non-places: Introduction to an Anthropology of Supermodernity translated by J Howe (Verso, London)

Baberg T W, 2001, "Man-machine-interface in modern transport systems from an aviation safety perspective" Aerospace Science and Technology 5 495-504

Batty M, 1997a, "The computable city” International Planning Studies 2 155-173

Batty M, 1997b, "Virtual geography" Futures 29337 - 352

Bradsher K, 2003, "Globetrotting traveler infected with SARS" The New York Times 10 April, http://www.nytimes.com/2003/04/10/science/sciencespecial/10CND-SARS.html

Butler D L, 2001, "Technogeopolitics and the struggle for control of world air routes, 1910 - 1928" Political Geography $20635-658$

Castells M, 1996 Rise of the Network Society (Blackwell, Oxford)

Castells M, 1998 End of the Millennium (Blackwell, Oxford)

Cosgrove D, 1994, "Contested global visions: one-world, whole-Earth and the Apollo space photographs" Annals of the Association of American Geographer 82 270-294

Crang M, 2002, "Between places: producing hubs, flows, and networks" Environment and Planning $A$ $34569-574$

Curry M, 1998 Digital Places: Living with Geographic Information Technologies (Routledge, London)

Deleuze G, Guattari F, 1987 A Thousand Plateaus: Capitalism and Schizophrenia translated by B Massumi (University of Minnesota Press, Minneapolis, MN)

Edwards P N, 1996 The Closed World: Computers and the Politics of Discourse in Cold War America (MIT Press, Cambridge, MA)

Everett R R, Zraket C A, Benington H D, 1983, "SAGE: a data processing system for air defense" IEEE Annals of the History of Computing 5330 - 339

Fleming D K, 1984, "Cartographic strategies for airline advertising" The Geographical Review 74 $76-93$

Foucault M, 1977 Discipline and Punish: The Birth of the Prison (Penguin Books, Harmondsworth, Middx)

Foucault M, 1978 The History of Sexuality (Penguin Books, Harmondsworth, Middx)

Gottdiener M, 2001 Life in the Air: Surviving the New Culture of Air Travel (Rowman and Littlefield, Totowa, NJ)

Graham B, 1995 Geography and Air Transport (John Wiley, Chichester, Sussex)

Graham S, 1998, "Spaces of surveillant simulation: new technologies, digital representations, and material geographies" Environment and Planning D: Society and Space 16483 - 503 
Graham S, Marvin S, 2001 Splintering Urbanism: Networked Infrastructures, Technological Mobilities and the Urban Condition (Routledge, London)

Graham S, Wood D, 2003, "Digitizing surveillance: categorization, space, inequality" Critical Social Policy $23227-248$

Harvey D, 1989 The Condition of Postmodernity (Basil Blackwell, Oxford)

Holland J H, 1998 Emergence: From Chaos to Order (Oxford University Press, Oxford)

IPCC, 1999 Aviation and the Global Environment special report from the Intergovernmental Panel on Climate Change, June 1999, Geneva, http://www.ipcc.ch/pub/av(E).pdf

Lessig L, 1999 Code and Other Laws of Cyberspace (Basic Books, New York)

Lyon D, 2003, "Airports as data filters: converging surveillance systems after September 11" Information, Communication and Ethics in Society $113-20$

MacPherson M, 1998 The Black Box (HarperCollins, New York)

Mitchell W J, 1996 City of Bits: Space, Place and the Infobahn (MIT Press, Cambridge, MA)

Parker K W, 2002, "Making connections: travel, technology, and global air travel networks" Social Change in the 21st Century Conference Queensland University of Technology, November 2002, http://www.socialchange.qut.edu.au/conference/docs/Conf\%20Papers/ParkerKen.pdf

Pehrson R J, 1996, "Software development for the Boeing 777" CrossTalk, The Journal of Defense Software Engineering January, http://www.www.stsc.hill.af.mil/crosstalk/1996/01/Boein777.asp

Risks List, 2003, "Computer error grounds Japanese flights" Forum on Risk to the Public in Computers and Related Systems 3 March 22 60, http://catless.ncl.ac.uk/Risks/22.60.html

Rolfe J M, Staples K J, 1986 Flight Simulation (Cambridge University Press, Cambridge)

Salkever A, 2002, "The intensifying scrutiny at airports" BusinessWeek 5 June, http://www.businessweek.com/technology/content/jun2002/tc2002065_2255.htm

Sampson A, 1984 Empires of the Sky: The Politics, Contests and Cartels of World Airlines (Hodder and Stoughton, London)

Smith D A, Timberlake M F, 2001, "World city networks and hierarchies, 1977 - 1997: an empirical analysis of global air travel links" American Behavioral Scientist 441656 - 1677

Thrift N, French S, 2002, "The automatic production of space" Transactions of the Institute of British Geographers, New Series $27309-335$

Van Zandt J P, 1944 The Geography of World Air Transport (The Brookings Institution, Washington, DC)

Waldrop M M, 1994 Complexity: The Emerging Science at the Edge of Order and Chaos (Penguin Books, Harmondsworth, Middx)

Weber M, Williams G, 2001, "Drivers of long-haul air transport route development" Journal of Transport Geography $9243-254$

Whitelegg J, Williams N, 2001 The Plane Truth: Aviation and the Environment The Ashden Trust, London, http://www.aef.org.uk/PDFs/5389SainsburyDoc.pdf

Yach D, Bettcher D, 1998, "The globalization of public health, I: threats and opportunities" American Journal of Public Health $\mathbf{8 8} 735-738$ 